\title{
NORMAL LATTICES AND COSEPARATION OF LATTICES
}

\author{
BARRY B. MITTAG \\ Department of Mathematics \\ Sacred Heart University \\ 5151 Park Avenue \\ Fairfield, CT 06432-1000 U.S.A.
}

(Received September 19, 1995)

\begin{abstract}
Let $X$ be an arbitrary non-empty set, and let $\mathcal{L}$ be a lattice of subsets of $X$ such that $\emptyset$, $X \in \mathcal{L}$. We first summarize a number of known conditions which are equivalent to $\mathcal{L}$ being normal. We then develop new equivalent conditions in terms of set functions associated with $\mu \in I(\mathcal{L})$, the set of all non-trival, zero-one valued, tintely additive measures on the algebra generated by $\mathcal{L}$. We tinally generalize all the above to the situation where $\mathcal{L}_{1}$ and $\mathcal{L}_{2}$ are a pair of lattices of subsets of $X$ with $\mathcal{L}_{1}^{\prime} \subset \mathcal{L}_{2}$, and where we obtain equivalent conditions for $\mathcal{L}_{1}$ to coseparate $\mathcal{L}_{2}$.
\end{abstract}

KEY WORDS AND PHRASES: Normal lattice, coseparation of lattices, zero-one valued measures, associated outer measures.

AMS SUBJECT CLASSIFICATION CODES: 28C15, 28A12.

\section{INTRODUCTION}

Let $X$ be an arbitrary non-empty set, and let $\mathcal{L}$ be a lattice of subsets of $X$ such that $\emptyset, X \in \mathcal{L}$.

Various necessary and sufficient conditions for the lattice $\mathcal{L}$ to be normal are known (see [4,5,6]), and we summarize a number of these in section 2 . We then give new necessary and sufficient conditions for the normality of $\mathcal{L}$ in section 3 . These conditions are in terms of set functions associated with a $\mu \in I(\mathcal{L})$, where $I(\mathcal{L})$ is the set of non-trivial, zero-one valued, finitely additive measures on the algebra generated by $\mathcal{L}$.

Section 4 is devoted to the more general situation of a pair of lattices $\mathcal{L}_{1}$ and $\mathcal{L}_{2}$ with $\mathcal{L}_{1} \subset \mathcal{L}_{2}$ and for which $\mathcal{L}_{1}$ coseparates $\mathcal{L}_{2}$. If $\mathcal{L}_{1}=\mathcal{L}_{2}$, then $\mathcal{L}_{1}$ coseparates itself if and only if it is normal. We proceed, at first, to give necessary and sufficient conditions for $\mathcal{L}_{1}$ to coseparate $\mathcal{L}_{2}$ which extend known necessary and sufficient conditions for normal lattices which are summarized in section 2 . Then we extend our new conditions for normality, to conditions both necessary and sufficient for $\mathcal{L}_{1}$ to coseparate $\mathcal{L}_{2}$ in terms of set functions associated with a $\mu \in I\left(\mathcal{L}_{1}\right)$.

We begin in section 2 with a brief summary of the notation and terminology used throughout the paper. Related matters can be found in $[2,4,6]$. We then turn our attention to normal lattices, and follow the program indicated above.

\section{BACKGROUND AND NOTATION}

Here we summarize briefly the notation and terminology that will be used throughout the paper Most of this is standard by now and follows that used in $[1,3,4,7]$ for example. We will also assume for convenience that all lattices considered contain the $\emptyset$ and $X$.

$X$ is an arbitrary non-empty set and $\mathcal{L}$ a lattice of subsets of $X . \mathcal{A}(\mathcal{L})$ denotes the algebra generated by $\mathcal{L}$, and $I(\mathcal{L})$ those non-trivial, finitely additive, zero-one valued measures defined on $\mathcal{A}(\mathcal{L}) . \quad I_{R}(\mathcal{L})$ 
denotes those $\mu \in I(\mathcal{L})$, which are $\mathcal{L}$-regular, i.e

$$
\mu(A)=\sup \{\mu(L) \mid L \subset A, L \in \mathcal{L}\}
$$

where $A \in \mathcal{A}(\mathcal{L})$. We note, that $\mu \in I_{R}(\mathcal{L})$ if and only if $\mu \in I(\mathcal{L})$, and

$$
\mu\left(L^{\prime}\right)=\sup \left\{\mu(\tilde{L}) \mid \tilde{L} \subset L^{\prime}, \tilde{L} \in \mathcal{L}\right\}
$$

where $L \in \mathcal{L}$. Here $L^{\prime}=X-L$, and we denote by $\mathcal{L}^{\prime}=\left\{L^{\prime} \mid L \in \mathcal{L}\right\} . \quad \mathcal{L}^{\prime}$ is the complementary lattice to $\mathcal{L}$.

We note that there exists a one-to-one correspondence between all prime $\mathcal{L}$-filters and $I(\mathcal{L})$ given by associating with $\mu \in I(\mathcal{L})$ the prime $\mathcal{L}$-filter

$$
\mathcal{F}=\{L \in \mathcal{L} \mid \mu(L)=1\} .
$$

Similarly, there exists a one-to-one correspondence between all $\mathcal{L}$-ultrafilters and $I_{R}(\mathcal{L})$ given by the same collection as in (2.1) only now $\mu \in I_{R}(\mathcal{L})$.

Finally, we note that if $\mathcal{H}$ is any collection of sets of $\mathcal{L}$ with the finite intersection property, i.e. the intersection of any finite number of sets of $\mathcal{H}$ is non-empty, then there exists a $\mu \in I_{R}(\mathcal{L})$ such that $\mu(A)=1$ for all $A \in \mathcal{H}$.

$I_{\sigma}(\mathcal{L})$ denotes those $\mu \in I(\mathcal{L})$ such that $\mu$ is $\sigma$-smooth on $\mathcal{L}$, i.e. if $L_{n} \in \mathcal{L}$ and $L_{n} \downarrow \emptyset$ then $\mu\left(L_{n}\right) \rightarrow 0$. There is a one-to-one correspondence between $I_{\sigma}(\mathcal{L})$ and all prime $\mathcal{L}$-filters with the countable intersection property.

$I^{\sigma}(\mathcal{L})$ denotes these $\mu \in I(\mathcal{L})$ that are $\sigma$-smooth on $\mathcal{A}(\mathcal{L})$, or, equivalently, are countably additive.

$I_{R}^{\sigma}(\mathcal{L})=I_{\sigma}(\mathcal{L}) \cap I_{R}(\mathcal{L})$, and it is easy to see that if $\mu \in I_{R}^{\sigma}(\mathcal{L})$ then $\mu \in I^{\sigma}(\mathcal{L})$.

If $\mu \in I(\mathcal{L})$, we denote by $\mu^{\prime}$ the following set function defined on $\mathcal{P}(X)$ : for $E \subset X$,

$$
\mu^{\prime}(E)=\inf \left\{\mu\left(L^{\prime}\right) \mid E \subset L^{\prime}, L \in \mathcal{L}\right\} .
$$

$\mu^{\prime}$ is a finitely subadditive outer measure.

If $\mu, \nu$ are set functions defined on $\mathcal{L}$, we write $\mu \leq \nu(\mathcal{L})$ if $\mu(L) \leq \nu(L)$ for all $L \in \mathcal{L}$. It is now clear that

$$
\mu \in I_{R}(\mathcal{L}) \text { if and only if } \quad \mu=\mu^{\prime}(\mathcal{L}) .
$$

A set function defined on $\mathcal{L}$ is called modular if $\nu\left(L_{1} \cup L_{2}\right)+\nu\left(L_{1} \cap L_{2}\right)=\nu\left(L_{1}\right)+\nu\left(L_{2}\right)$, for all $L_{1}, L_{2} \in \mathcal{L}$. If $\nu\left(L_{1} \cup L_{2}\right)+\nu\left(L_{1} \cap L_{2}\right) \leq \nu\left(L_{1}\right)+\nu\left(L_{2}\right)$ for all $L_{1}, L_{2} \in \mathcal{L}$, then $\nu$ is called submodular, and supermodular if the inequality is reversed.

We recall that $\mathcal{L}$ is countably compact (c.c.) if and only if $I(\mathcal{L})=I_{\sigma}(\mathcal{L})^{\prime}$, or, equivalently, if and only if $I_{R}(\mathcal{L})=I_{R}^{\sigma}(\mathcal{L})$.

$\mathcal{L}$ is countably paracompact (c.p.) if $A_{n} \downarrow \emptyset, A_{n} \in \mathcal{L}$ implies there exists $B_{n} \in \mathcal{L}, A_{n} \subset B_{n}^{\prime} \downarrow \emptyset$. Clearly if $\mathcal{L}$ is c.p. then $I_{\sigma}\left(\mathcal{L}^{\prime}\right) \subset I_{\sigma}(\mathcal{L})$.

$\mathcal{L}$ is a normal lattice if for any $A_{1}, A_{2} \in \mathcal{L}$ with $A_{1} \cap A_{2}=\emptyset$ there exist $B_{1}, B_{2} \in \mathcal{L}$ with $A_{1} \subset B_{1}^{\prime}, A_{2} \subset B_{2}^{\prime}$ and $B_{1}^{\prime} \cap B_{2}^{\prime}=\emptyset$.

We summarize some equivalent characterizations of normality in the following theorem (see $[4,5,6])$.

THEOREM 2.1. $\mathcal{L}$ is normal (where $\emptyset, X \in \mathcal{L}$ ) is equivalent to any of the following:

1) For each $\mu \in I(\mathcal{L})$, there exists a unique $\nu \in I_{R}(\mathcal{L})$ such that $\mu \leq \nu(\mathcal{L})$.

2) For any $\mu \in I(\mathcal{L})$ and $\nu \in I_{R}(\mathcal{L})$ such that $\mu \leq \nu(\mathcal{L})$ then $\mu \leq \nu=\nu^{\prime}=\mu^{\prime}(\mathcal{L})$.

3) If $\mu \leq \nu(\mathcal{L})$ where $\mu \in I(\mathcal{L}), \nu \in I_{R}(\mathcal{L})$ then $\nu\left(L^{\prime}\right)=\sup \left\{\mu(\tilde{L}) \mid \tilde{L} \subset L^{\prime}, \tilde{L} \in \mathcal{L}\right\}$ where $L \in \mathcal{L}$

4) If $L \subset L_{1}^{\prime} \cup L_{2}^{\prime}$ where $L, L_{1}, L_{2} \in \mathcal{L}$, then $L=A \cup B$ where $A, B \in \mathcal{L}$ and $A \subset L_{1}^{\prime}, B \subset L_{2}^{\prime}$.

5) For any $\mu \in I(\mathcal{L}), \mathcal{F}=\left\{L \in \mathcal{L} \mid \mu^{\prime}(L)=1\right\}$ is an $\mathcal{L}$-ultrafilter.

Further characterizations of normality will be developed in section 3 . We just note one consequence of normality. We denote by $I_{w}(\mathcal{L})$ those $\mu \in I(\mathcal{L})$ such that $\mu\left(L^{\prime}\right)=1$ implies there exists an $\tilde{L} \in \mathcal{L}$, 
$\tilde{L} \subset L^{\prime}$, and $\mu^{\prime}(\tilde{L})=1$ where $L \in \mathcal{L}$. If $\mathcal{L}$ is normal, then $I_{w}(\mathcal{L})=I_{R}(\mathcal{L})$. The converse, however, is not true in general.

If we have a pair of lattices, $\mathcal{L}_{1}, \mathcal{L}_{2}$ of subsets of $X$ with $\mathcal{L}_{1} \subset \mathcal{L}_{2}$, and if $\mu \in I\left(\mathcal{L}_{2}\right)$ then its restriction to $\mathcal{A}\left(\mathcal{L}_{1}\right)$ will be denoted by $\left.\mu\right|_{\mathcal{L}_{1}}$ or simply $\mu \mid$ if there is no ambiguity.

In general if we have a pair of lattices $\mathcal{L}_{1}, \mathcal{L}_{2}$ we define:

$\mathcal{L}_{1}$ semiseparates $\mathcal{L}_{2}$ if for all $A \in \mathcal{L}_{1}, B \in \mathcal{L}_{2}$ such that $A \cap B=\emptyset$, there exists an $A_{1} \in \mathcal{L}_{1}$ such that $B \subset A_{1}$ and $A \cap A_{1}=\emptyset$.

$\mathcal{L}_{1}$ separates $\mathcal{L}_{2}$ if for all $B_{1}, B_{2} \in \mathcal{L}_{2}$ such that $B_{1} \cap B_{2}=\emptyset$, there exists $A_{1}, A_{2} \in \mathcal{L}_{1}$ such that $B_{1} \subset A_{1}, B_{2} \subset A_{2}$ and $A_{1} \cap A_{2}=\emptyset$.

$\mathcal{L}_{1}$ coseparates $\mathcal{L}_{2}$ if for all $B_{1}, B_{2} \in \mathcal{L}_{2}$ such that $B_{1} \cap B_{2}=\emptyset$, there exists $A_{1}, A_{2} \in \mathcal{L}_{1}$ such that $B_{1} \subset A_{1}^{\prime}, B_{2} \subset A_{2}^{\prime}$ and $A_{1}^{\prime} \cap A_{2}^{\prime}=\emptyset$.

$\mathcal{L}_{2}$ coallocates $\mathcal{L}_{1}$ if $A \subset B_{1}^{\prime} \cup B_{2}^{\prime}$, where $A \in \mathcal{L}_{1}, B_{1}, B_{2} \in \mathcal{L}_{2}$, then there exists $A_{1}, A_{2} \in \mathcal{L}_{1}$ such that $A=A_{1} \cup A_{2}, A_{1} \subset B_{1}^{\prime}$ and $A_{2} \subset B_{2}^{\prime}$.

Under our assumption that all lattices involved contain $\emptyset, X$ and assuming that $\mathcal{L}_{1} \subset \mathcal{L}_{2}$, then $\mathcal{L}_{1}$ coseparates $\mathcal{L}_{2}$ if and only if $\mathcal{L}_{2}$ coallocates $\mathcal{L}_{1}$.

Finally, if $\nu$ is a finite outer measure (either finitely subadditive or countably subadditive) defined on $P(X)$, we denote by $\mathcal{S}_{\nu}$ the $\nu$-measurable sets, so

$$
\mathcal{S}_{\nu}=\left\{E \subset X \mid \nu(G)=\nu(G \cap E)+\nu\left(G \cap E^{\prime}\right) \text { for all } G \subset X\right\} .
$$

$\nu$ is regular if for any $A \subset X$, there exists an $E \in \mathcal{S}_{\nu}$ with $A \subset E$ and $\nu(A)=\nu(E)$. If $\nu$ is a regular outer measure which is finite then

$$
\mathcal{S}_{\nu}=\left\{E \subset X \mid \nu(X)=\nu(E)+\nu\left(E^{\prime}\right)\right\} .
$$

If $\nu$ is any outer measure that just assumes the values zero and one, then $\nu$ is clearly regular.

\section{NORMAL LATTICES}

In this section we wish to get characterizations of normal lattices in terms of certain set functions associated with a $\mu \in I(\mathcal{L})$. In the presence of normality, these set functions have been investigated $[2,4,5,6]$. We will summarize briefly these results, but we wish to go beyond this, and show that properties of these set functions can be utilized to give necessary and sufficient conditions for a lattice to be normal.

DEFINITION 3.1. Let $\mu \in I(\mathcal{L})$ and let $E \subset X$.

a) $\mu_{2}(E)=\sup \{\mu(L) \mid L \subset E, L \in \mathcal{L}\}$.

b) $\bar{\mu}(E)=\inf \left\{\mu_{\imath}\left(L^{\prime}\right) \mid E \subset L^{\prime}, L \in \mathcal{L}\right\}$.

It follows readily from the definition that, for $\mu \in I(\mathcal{L})$,

$$
\begin{aligned}
& \mu_{\imath}=\mu \leq \bar{\mu} \leq \mu^{\prime}(\mathcal{L}), \quad \text { and } \\
& \bar{\mu}=\mu_{\imath} \leq \mu=\mu^{\prime}\left(\mathcal{L}^{\prime}\right) .
\end{aligned}
$$

Now, we have

THEOREM 3.1. If $\mathcal{L}$ is a normal lattice and if $\mu \in I(\mathcal{L})$, then

a) $\mu_{i}$ is finitely additive and finitely subadditive on $\mathcal{L}^{\prime}$.

b) $\bar{\mu}$ is a finitely subadditive outer measure.

PROOF. a) Let $A, B \in \mathcal{L}$, and suppose $\mu_{i}\left(A^{\prime} \cup B^{\prime}\right)=1$. Then there exists $L \in \mathcal{L}$ such that $L \subset A^{\prime} \cup B^{\prime}$ and $\mu(L)=1$. From Theorem 2.1 part 4 of section 2, it follows that there exists $A_{1}, B_{1} \in \mathcal{L}$ with $L=A_{1} \cup B_{1}, A_{1} \subset A^{\prime}, B_{1} \subset B^{\prime}$. Thus $\mu(L) \leq \mu\left(A_{1}\right)+\mu\left(B_{1}\right)$, consequently, $\mu\left(A_{1}\right)=1$ or $\mu\left(B_{1}\right)=1$. By (3.1) this implies that $\mu_{2}\left(A_{1}\right)=1$ or $\mu_{2}\left(B_{1}\right)=1$ so $\mu_{2}\left(A^{\prime}\right)=1$ or $\mu_{2}\left(B^{\prime}\right)=1$ since $\mu_{2}$ is monotone. It is now clear that $\mu_{i}\left(A^{\prime} \cup B^{\prime}\right) \leq \mu_{i}\left(A^{\prime}\right)+\mu_{i}\left(B^{\prime}\right)$ for any $A$, $B \in \mathcal{L}$. Again, if $\mu_{2}\left(A^{\prime} \cup B^{\prime}\right)=1$ and if $A^{\prime} \cap B^{\prime}=\emptyset, A, B \in \mathcal{L}$, then using the previous notation 
$A_{1} \cap B_{1}=\emptyset$, and $1=\mu(L)=\mu\left(A_{1} \cup B_{1}\right)=\mu\left(A_{1}\right)+\mu\left(B_{1}\right)$. Say, $\mu\left(A_{1}\right)=1$ and $\mu\left(B_{1}\right)=0$, and clearly $\mu_{i}\left(A^{\prime}\right)=1$ and $\mu_{i}\left(B^{\prime}\right)=0$ from which the additivity of $\mu_{2}$ on $\mathcal{L}^{\prime}$ follows.

b) Clearly $\bar{\mu}(\emptyset)=0, \bar{\mu}$ is monotone, and all we need prove is the finite subadditivity of $\bar{\mu}$. Let $E_{1}$ and $E_{2}$ be arbitrary subsets with $\bar{\mu}\left(E_{1} \cup E_{2}\right)=1$. Then $\mu_{2}\left(L^{\prime}\right)=1$ for all $L \supset E_{1} \cup E_{2}, L \in \mathcal{L}$. If both $\bar{\mu}\left(E_{1}\right)=0$ and $\bar{\mu}\left(E_{2}\right)=0$, then there exists $L_{1}^{\prime}, L_{2}^{\prime} \in \mathcal{L}^{\prime}$ with $L_{1}^{\prime} \supset E_{1}, L_{2}^{\prime} \supset E_{2}$ and $\mu_{i}\left(L_{1}^{\prime}\right)=\mu_{i}\left(L_{2}^{\prime}\right)=0$. Then $L_{1}^{\prime} \cup L_{2}^{\prime} \supset E_{1} \cup E_{2}$ and $\mu_{i}\left(L_{1}^{\prime} \cup L_{2}^{\prime}\right)=0$ by part a), which is a contradiction, and completes the proof.

Since $\bar{\mu}$ is a finitely subadditive outer measure, we denote by $\mathcal{S}_{\bar{\mu}}$, the $\bar{\mu}$-measurable sets, i.e.

$$
\mathcal{S}_{\bar{\mu}}=\left\{E \subset X \mid \bar{\mu}(G)=\bar{\mu}(G \cap E)+\bar{\mu}\left(G \cap E^{\prime}\right) \text { for all } G \subset X\right\} .
$$

Clearly $\mathcal{S}_{\bar{\mu}}$ is an algebra, and since $\mu$ and, therefore, $\bar{\mu}$ just assume the values 0 and 1 , we have

$$
\mathcal{S}_{\bar{\mu}}=\left\{E \subset X \mid \bar{\mu}(X)=\bar{\mu}(E)+\bar{\mu}\left(E^{\prime}\right)\right\} .
$$

\section{Now we show}

THEOREM 3.2. If $\mathcal{L}$ is normal and if $\mu \in I(\mathcal{L})$, then $\mathcal{A}(\mathcal{L}) \subset \mathcal{S}_{\bar{\mu}}$.

PROOF. We need only show that if $L \in \mathcal{L}$, then $\bar{\mu}(X) \geq \bar{\mu}(L)+\bar{\mu}\left(L^{\prime}\right)$. Suppose $\bar{\mu}\left(L^{\prime}\right)=1$. By (3.1), this implies $\mu_{2}\left(L^{\prime}\right)=1$. Hence, there exists $\tilde{L} \in \mathcal{L}, \tilde{L} \subset L^{\prime}$ and $\mu(\tilde{L})=1$. Since $\tilde{L} \cap L=\emptyset$, and since $\mathcal{L}$ is normal, there exists $B_{1}^{\prime}, B_{2}^{\prime} \in \mathcal{L}^{\prime}$ with $L \subset B_{1}^{\prime}, \tilde{L} \subset B_{2}^{\prime}$ and $B_{1}^{\prime} \cap B_{2}^{\prime}=\emptyset$. Clearly, $\mu\left(B_{2}^{\prime}\right)=1$ and $\mu\left(B_{1}^{\prime}\right)=0$. Thus $\bar{\mu}\left(B_{1}^{\prime}\right)=0$ by (3.1), and $\bar{\mu}(L)=0$. Thus $\bar{\mu}\left(L^{\prime}\right)$ and $\bar{\mu}(L)$ can't both be one, which completes the proof.

Finally we have

THEOREM 3.3. If $\mathcal{L}$ is normal and if $\mu \in I(\mathcal{L})$ then

a) $\left.\bar{\mu}\right|_{\mathcal{A}(\mathcal{L})} \in I_{R}(\mathcal{L})$, and

b) $\bar{\mu}=\mu^{\prime}(\mathcal{L})$.

PROOF. a) Since $\bar{\mu}$ is a finitely additive measure on $\mathcal{S}_{\bar{\mu}}$, it follows that $\left.\bar{\mu}\right|_{\mathcal{A}(\mathcal{L})} \in I(\mathcal{L})$.

Also, for $L \in \mathcal{L}$,

$$
\bar{\mu}^{\prime}(L)=\inf \left\{\bar{\mu}\left(A^{\prime}\right) \mid L \subset A^{\prime}, A \in \mathcal{L}\right\},
$$

but $\bar{\mu}=\mu_{2}\left(\mathcal{L}^{\prime}\right)$. Thus $\bar{\mu}^{\prime} \overline{\overline{\mathcal{L}}} \bar{\mu}$, therefore, $\left.\bar{\mu}\right|_{\mathcal{A}(\mathcal{L})} \in I_{R}(\mathcal{L})$ (see section 2).

b) Since $\mu \leq \bar{\mu}(\mathcal{L})$, by a) and by normality (see section 2 ), $\mu \leq \bar{\mu}=\bar{\mu}^{\prime}=\mu^{\prime}(\mathcal{L})$.

Let $\nu$ be a set function defined on all subsets of $X$. Recall $\nu$ is submodular if and only if

$$
\nu\left(E_{1} \cup E_{2}\right)+\nu\left(E_{1} \cap E_{2}\right) \leq \nu\left(E_{1}\right)+\nu\left(E_{2}\right)
$$

for all $E_{1}, E_{2} \subset X$.

It is easy to see that the following holds.

LEMMA 3.4. If $\nu$ is a monotone set function defined on all sets $E \subset X$ that assumes only the values 0 and 1 , then $\nu$ is finitely subadditive if and only if $\nu$ is submodular.

Now we establish:

THEOREM 3.5. If $\mu \in I(\mathcal{L})$, then $\mathcal{L}$ is normal if and only if $\bar{\mu}$ is submodular (or equivalently if and only if $\bar{\mu}$ is a finitely subadditive outer measure)

PROOF. If $\mathcal{L}$ is normal, then $\bar{\mu}$ is a finitely subadditive outer measure by Theorem $3.1 \mathrm{~b}$ ), and, therefore, submodular by the Lemma.

Conversely, suppose $\bar{\mu}$ is submodular. If $\mathcal{L}$ is not normal, then there exists $A_{1}, A_{2} \in \mathcal{L}$ such that $A_{1} \cap A_{2}=\emptyset$, but for all $B_{1}^{\prime}, B_{2}^{\prime} \in \mathcal{L}^{\prime}$ with $A_{1} \subset B_{1}^{\prime}, A_{2} \subset B_{2}^{\prime}$, we have $B_{1}^{\prime} \cap B_{2}^{\prime} \neq \emptyset$.

This implies that the set

$$
\mathcal{B}=\left\{B^{\prime} \in \mathcal{L}^{\prime} \mid B^{\prime} \supset A_{1} \text { or } B^{\prime} \supset A_{2}\right\}
$$


has the finite intersection property. Consequently there exists a $\mu \in I_{R}(\mathcal{L})$ such that $\mu\left(B^{\prime}\right)=1$ for all $B^{\prime} \in \mathcal{B}$. Hence, $\mu(B)=0$ for all $B^{\prime} \in \mathcal{B}$, which implies $\mu_{2}\left(A_{1}^{\prime}\right)=0$ and $\mu_{2}\left(A_{2}^{\prime}\right)=0$. Thus, $\bar{\mu}\left(A_{1}^{\prime}\right)=\bar{\mu}\left(A_{2}^{\prime}\right)=0$ by (3.1). But $1=\bar{\mu}(X)=\bar{\mu}\left(A_{1}^{\prime} \cup A_{2}^{\prime}\right)=0$ which is a contradiction since $\bar{\mu}$ is submodular.

As our characterization theorem, we have

THEOREM 3.6. If $\mu \in I(\mathcal{L})$, then $\mathcal{L}$ is normal if and only if $\bar{\mu}=\mu^{\prime}(\mathcal{L})$.

PROOF. If $\mathcal{L}$ is normal then $\bar{\mu}=\mu^{\prime}(\mathcal{L})$ by Theorem 3.3 part b).

Conversely, suppose $\bar{\mu}=\mu^{\prime}(\mathcal{L})$ and $\mathcal{L}$ is not normal. Then using the same notation and construction as in Theorem 3.5, we have $\mu_{\imath}\left(A_{2}^{\prime}\right)=0$, while $A_{1} \subset B^{\prime}, B \in \mathcal{L}$ implies $\mu\left(B^{\prime}\right)=1$, but $A_{1} \subset A_{2}^{\prime}$. Thus $\bar{\mu}\left(A_{1}\right)=0$, while clearly $\mu^{\prime}\left(A_{1}\right)=1$. This contradiction proves the theorem.

\section{COSEPARATION OF LATTICES}

In the present section, we will extend the results of sections 2 and 3 on normal lattices to a pair of lattices $\mathcal{L}_{1}$ and $\mathcal{L}_{2}$ such that $\mathcal{L}_{1} \subset \mathcal{L}_{2}$, and where $\mathcal{L}_{1}$ coseparates $\mathcal{L}_{2}$. Clearly, if $\mathcal{L}_{1}=\mathcal{L}_{2}$ then $\mathcal{L}_{1}$ coseparates itself if and only if it is normal. The work done here also extends that done in $[2,5,6]$.

Our first result directly generalizes Theorem 2.1 part 1 ).

THEOREM 4.1. Let $\mathcal{L}_{1}$ and $\mathcal{L}_{2}$ be lattices of subsets of $X$ such that $\mathcal{L}_{1} \subset \mathcal{L}_{2}$. Then $\mathcal{L}_{1}$ coseparates $\mathcal{L}_{2}$ if and only if for any $\mu \in I\left(\mathcal{L}_{1}\right)$ and any $\nu_{1}, \nu_{2} \in I_{R}\left(\mathcal{L}_{2}\right)$ such that $\mu \leq \nu_{1}\left(\mathcal{L}_{1}\right)$ and $\mu \leq \nu_{2}\left(\mathcal{L}_{1}\right)$ then $\nu_{1}=\nu_{2}$.

PROOF. 1) We assume that $\mathcal{L}_{1}$ coseparates $\mathcal{L}_{2}$. If $\nu_{1} \neq \nu_{2}$ then there exists $B_{1}, B_{2} \in \mathcal{L}_{2}$ such that $\nu_{1}\left(B_{1}\right)=1, \nu_{2}\left(B_{2}\right)=1$, and $B_{1} \cap B_{2}=\emptyset$. Hence, there exists $A_{1}, A_{2} \in \mathcal{L}_{1}$ with $B_{1} \subset A_{1}^{\prime}, B_{2} \subset A_{2}^{\prime}$ and $A_{1}^{\prime} \cap A_{2}^{\prime}=\emptyset$. Consequently, $A_{1} \cup A_{2}=X$ so $\mu\left(A_{1}\right)=1$ or $\mu\left(A_{2}\right)=1$. But $A_{1} \subset B_{1}^{\prime}$, and $\nu_{1}\left(B_{1}^{\prime}\right)=0$, so $\nu_{1}\left(A_{1}\right)=0$; hence $\mu\left(A_{1}\right)=0$. Similarly $A_{2} \subset B_{2}^{\prime}$, and $\nu_{2}\left(B_{2}^{\prime}\right)=0$ so $\mu\left(A_{2}\right)=0$, from this contradiction, we conclude that $\nu_{1}=\nu_{2}$.

2) Conversely, assuming the condition of the theorem holds, if $\mathcal{L}_{1}$ does not coseparate $\mathcal{L}_{2}$ then there exists $B_{1}, B_{2} \in \mathcal{L}_{2}$ such that

$$
\mathcal{H}=\left\{A^{\prime} \in \mathcal{L}_{1}^{\prime} \mid B_{1} \subset A^{\prime} \text { or } B_{2} \subset A^{\prime}\right\}
$$

has the finite intersection property. Therefore, there exists a $\mu \in I\left(\mathcal{L}_{1}\right)$ such that $\mu\left(A^{\prime}\right)=1$ for all $A^{\prime} \in \mathcal{H}$. Now let $L_{1} \in \mathcal{L}_{1}$ with $\mu\left(L_{1}\right)=1$. Then $\mu\left(L_{1}^{\prime}\right)=0$ so $L_{1}^{\prime} \notin \mathcal{H}$. Hence, $L_{1} \cap B_{1} \neq \emptyset$ and $L_{1} \cap B_{2} \neq \emptyset$ for all $L_{1} \in \mathcal{L}_{1}$ with $\mu\left(L_{1}\right)=1$. Thus if we let

$$
\mathcal{H}_{1}=\left\{L_{2} \in \mathcal{L}_{2} \mid L_{2} \supset L_{1} \cap B_{1}, L_{1} \in \mathcal{L}_{1} \text { with } \mu\left(L_{1}\right)=1\right\} \text {, }
$$

and

$$
\mathcal{H}_{2}=\left\{L_{2} \in \mathcal{L}_{2} \mid L_{2} \supset L_{1} \cap B_{2}, L_{1} \in \mathcal{L}_{1} \text { with } \mu\left(L_{1}\right)=1\right\}
$$

then $\mathcal{H}_{1}$ and $\mathcal{H}_{2}$ are $\mathcal{L}_{2}$-filters Consequently, there exists $\nu_{1}, \nu_{2} \in I_{R}\left(\mathcal{L}_{2}\right)$ such that $\nu_{1}\left(L_{2}\right)=1$ for all $L_{2} \in \mathcal{H}_{1}$ and $\nu_{2}\left(L_{2}\right)=1$ for all $L_{2} \in \mathcal{H}_{2} . \quad \nu_{1} \neq \nu_{2}$ since $B_{1} \in \mathcal{H}_{1}$ and $B_{2} \in \mathcal{H}_{2}$, and $B_{1} \cap B_{2}=\emptyset$. Also $\mu \leq \nu_{1}\left(\mathcal{L}_{1}\right)$ since, if $A \in \mathcal{L}_{1}$ and $\mu(A)=1$ then $A \in \mathcal{H}$, clearly, so $\nu_{1}(A)=1$; similarly $\mu \leq \nu_{2}\left(\mathcal{L}_{1}\right)$ which completes the proof.

The next theorem generalizes Theorem 2.1 part 2).

THEOREM 4.2. Let $\mathcal{L}_{1}$ and $\mathcal{L}_{2}$ be lattices of subsets of $X$ such that $\mathcal{L}_{1} \subset \mathcal{L}_{2}$. Then $\mathcal{L}_{1}$ coseparates $\mathcal{L}_{2}$ if and only if for any $\mu \in I\left(\mathcal{L}_{1}\right)$ and $\nu \in I_{R}\left(\mathcal{L}_{2}\right)$ such that $\mu \leq \nu\left(\mathcal{L}_{1}\right)$, we have $\nu=\mu^{\prime}\left(\mathcal{L}_{2}\right)$.

PROOF. 1) Suppose $\mathcal{L}_{1}$ coseparates $\mathcal{L}_{2}$. Clearly $\nu^{\prime} \leq \mu^{\prime}$. Suppose $\nu\left(L_{2}\right)=0$ where $L_{2} \in \mathcal{L}_{2}$. Then $\nu\left(L_{2}^{\prime}\right)=1$; hence $L_{2}^{\prime} \supset \tilde{L}_{2} \in \mathcal{L}_{2}$ and $\nu\left(\tilde{L}_{2}\right)=1$. Now $L_{2} \cap \tilde{L}_{2}=\emptyset$ so there exists $L_{1}, \tilde{L}_{1} \in \mathcal{L}_{1}$, $\tilde{L}_{2} \subset \tilde{L}_{1}^{\prime}, L_{2} \subset L_{1}^{\prime}$ and $L_{1}^{\prime} \cap \tilde{L}_{1}^{\prime}=\emptyset$, so $\nu\left(\tilde{L}_{1}^{\prime}\right)=1$, and, therefore, $\mu\left(\tilde{L}_{1}^{\prime}\right)=1$. Consequently, $\mu\left(L_{1}^{\prime}\right)=0$, so $\mu^{\prime}\left(L_{2}\right)=0$. This implies that 


$$
\nu=\nu^{\prime}=\mu^{\prime}\left(\mathcal{L}_{2}\right)
$$

Conversely, if this condition holds, and if $\mu \in I\left(\mathcal{L}_{1}\right)$, and $\nu_{1}, \nu_{2} \in I_{R}\left(\mathcal{L}_{2}\right)$ with $\mu \leq \nu_{1}\left(\mathcal{L}_{1}\right), \mu \leq \nu_{2}\left(\mathcal{L}_{1}\right)$ then $\nu_{1}=\nu_{1}^{\prime}=\mu^{\prime}\left(\mathcal{L}_{2}\right)$ and $\nu_{2}=\nu_{2}^{\prime}=\mu^{\prime}\left(\mathcal{L}_{2}\right)$, so $\nu_{1}=\nu_{2}$, and $\mathcal{L}_{1}$ coseparates $\mathcal{L}_{2}$ by Theorem 4.1.

Note: Clearly Theorem 4.2 is equivalent to the following: Let $\mathcal{L}_{1} \subset \mathcal{L}_{2}$ be lattices of subsets of $X$, and let $\mu \in I\left(\mathcal{L}_{1}\right)$ and $\nu \in I_{R}\left(\mathcal{L}_{2}\right)$ be arbitrary with $\mu \leq \nu\left(\mathcal{L}_{1}\right)$. Then $\mathcal{L}_{1}$ coseparates $\mathcal{L}_{2}$ if and only if $\nu\left(B^{\prime}\right)=1, B \in \mathcal{L}_{2}$, there exists an $A \in \mathcal{L}_{1}$ with $A \subset B^{\prime}$, and $\mu(A)=1$. Clearly this result extends Theorem 2.1 part 3.

We now extend the comment following Theorem 2.1.

Theorem 4.3. Let $\mathcal{L}_{1}$ and $\mathcal{L}_{2}$ be lattices of subsets of $X$ such that $\mathcal{L}_{1} \subset \mathcal{L}_{2}$. Also let $\mathcal{L}_{1}$ coseparate $\mathcal{L}_{2}$. Then, if for $\mu \in I\left(\mathcal{L}_{1}\right)$ and $\nu \in I\left(\mathcal{L}_{2}\right)$ with $\mu \leq \nu\left(\mathcal{L}_{1}\right)$ and with $\nu\left(B^{\prime}\right)=\sup \left\{\mu^{\prime}(A) \mid A \subset B^{\prime}, A \in \mathcal{L}_{1}\right\}$, $B \in \mathcal{L}_{2}$, we have $\nu \in I_{R}\left(\mathcal{L}_{2}\right)$.

PROOF. Suppose $\nu\left(B^{\prime}\right)=1$, where $B \in \mathcal{L}_{2}$. Then there exists $A \in \mathcal{L}, A \subset B^{\prime}$ and $\mu^{\prime}(A)=1$. Since $\mathcal{L}_{1}$ coseparates $\mathcal{L}_{2}$ there exists $A_{1}, A_{2} \in \mathcal{L}_{1}$ with $A \subset A_{1}^{\prime}, B \subset A_{2}^{\prime}$, and $A_{1}^{\prime} \cap A_{2}^{\prime}=\emptyset$, so $A \subset A_{1}^{\prime} \subset A_{2} \subset B^{\prime}$. Thus $\mu^{\prime}\left(A_{1}^{\prime}\right)=1=\mu\left(A^{\prime}\right)$, so $\mu\left(A_{2}\right)=1$. Hence $\nu\left(A_{2}\right)=1$, and $A_{2} \in \mathcal{L}_{1} \subset \mathcal{L}_{2}$ so $\nu \in I_{R}\left(\mathcal{L}_{2}\right)$.

We recall:

DEFINITION 4.1. The lattice $\mathcal{L}$ is almost countably compact if $I_{R}\left(\mathcal{L}^{\prime}\right) \subset I_{\sigma}(\mathcal{L})$.

Clearly if $\mathcal{L}$ is countably compact then $\mathcal{L}$ is almost countably compact. While, if $\mathcal{L}$ is normal, countably paracompact and almost countably compact, then $\mathcal{L}$ is countably compact.

THEOREM 4.4. Suppose $\mathcal{L}_{1} \subset \mathcal{L}_{2}$ and $\mathcal{L}_{1}$ coseparates $\mathcal{L}_{2}$. If $\mu \in I_{\sigma}\left(\mathcal{L}_{1}\right)$ and $\nu \in I_{R}\left(\mathcal{L}_{2}\right)$ such that $\mu \leq \nu\left(\mathcal{L}_{1}\right)$ then $\nu \in I_{\sigma}\left(\mathcal{L}_{2}^{\prime}\right)$.

PROOF. If $\nu \notin I_{\sigma}\left(\mathcal{L}_{2}^{\prime}\right)$, then there exists a sequence $\left\{B_{n}^{\prime}\right\}, B_{n} \in \mathcal{L}_{2}$ such that $B_{n}^{\prime} \downarrow \emptyset$ and $\nu\left(B_{n}^{\prime}\right)=1$ for all $n$. By the note after Theorem 4.2 there exists $A_{n} \in \mathcal{L}_{1}$ with $A_{n} \subset B_{n}^{\prime}$ and $\mu\left(A_{n}\right)=1$ for all $n$. Clearly, we may assume $A_{n} \downarrow$, so $A_{n} \downarrow \emptyset$, which implies $\mu \notin I_{\sigma}\left(\mathcal{L}_{1}\right)$, a contradiction.

THEOREM 4.5. Let $\mathcal{L}_{1} \subset \mathcal{L}_{2}$ and $\mathcal{L}_{1}$ coseparates $\mathcal{L}_{2}$. If $\nu \in I_{R}\left(\mathcal{L}_{2}\right)$ and if $\mathcal{L}_{1}$ is almost countably compact then $\nu \in I_{\sigma}\left(\mathcal{L}_{2}^{\prime}\right)$.

PROOF. Let $\lambda=\left.\nu\right|_{\mathcal{A}\left(\mathcal{L}_{1}\right)} . \quad \lambda \in I_{R}\left(\mathcal{L}_{1}\right)$, and since $\mathcal{L}_{1}$ is almost countably compact, there exists a $\mu \in I_{\sigma}(\mathcal{L})$ such that

$$
\mu \leq \lambda\left(\mathcal{L}_{1}\right)
$$

Now, by Theorem 4.4, it follows that $\nu \in I_{\sigma}\left(\mathcal{L}_{2}^{\prime}\right)$, which completes the proof.

REMARK. Under the assumption of Theorem 4.5, if in addition, $I_{\sigma}\left(\mathcal{L}_{2}^{\prime}\right) \subset I_{\sigma}\left(\mathcal{L}_{2}\right)$, then $\nu \in I_{R}^{\sigma}\left(\mathcal{L}_{2}\right)$, in which case $\mathcal{L}_{2}$ is countably compact.

We now wish to extend the results of Theorems 3.1-3.3, to the situation of a pair of lattices $\mathcal{L}_{1}, \mathcal{L}_{2}$ with $\mathcal{L}_{1} \subset \mathcal{L}_{2}$. We define for $\mu \in I(\mathcal{L})$ and any $E \subset X$.

a) $\mu_{i}(E)=\sup \left\{\mu\left(L_{1}\right) \mid L_{1} \subset E, L_{1} \in \mathcal{L}_{1}\right\}$.

b) $\bar{\mu}_{i}(E)=\inf \left\{\mu_{i}\left(L_{2}^{\prime}\right) \mid E \subset L_{2}^{\prime}, L_{2} \in \mathcal{L}_{2}\right\}$.

Arguing analogously to the proofs in Theorems 3.1-3.3, we obtain readily,

THEOREM 4.6. Let $\mathcal{L}_{1} \subset \mathcal{L}_{2}$ and let $\mathcal{L}_{1}$ coseparate $\mathcal{L}_{2}$. Then, for $\mu \in I\left(\mathcal{L}_{1}\right)$,

a) $\mu_{2}$ is finitely additive and finitely subadditive on $\mathcal{L}_{2}$.

b) $\bar{\mu}$ is a finitely subadditive outer measure.

c) $\mathcal{A}\left(\mathcal{L}_{1}\right) \subset \mathcal{S}_{\bar{\mu}}$.

d) $\nu=\left.\bar{\mu}\right|_{\mathcal{A}\left(\mathcal{L}_{2}\right)} \in I_{R}\left(\mathcal{L}_{2}\right)$, and $\mu \leq \nu\left(\mathcal{L}_{1}\right)$.

Furthermore, adhering to the above notation, we have

THEOREM 4.7. $\mathcal{L}_{1}$ coseparates $\mathcal{L}_{2}$ if and only if

$$
\mathcal{G}_{\mu^{\prime}}=\left\{L_{2} \in \mathcal{L}_{2} \mid \mu^{\prime}\left(L_{2}\right)=1\right\}
$$


is a prime $\mathcal{L}_{2}$-filter for any $\mu \in I\left(\mathcal{L}_{1}\right)$.

PROOF. 1) If $\mathcal{L}_{1}$ coseparates $\mathcal{L}_{2}$ then $\nu=\nu^{\prime}=\mu^{\prime}\left(\mathcal{L}_{2}\right)$ since $\nu \in I_{R}\left(\mathcal{L}_{2}\right)$ and using Theorem 4.2. From this it follows immediately that $\mu^{\prime}$ is modular on $\mathcal{L}_{2}$ and $\mathcal{G}_{\mu^{\prime}}$ is a prime $\mathcal{L}_{2}$-filter.

2) Suppose for any $\mu \in I\left(\mathcal{L}_{1}\right), \mathcal{G}_{\mu^{\prime}}$ is a prime $\mathcal{L}_{2}$-filter. If $\mathcal{L}_{1}$ does not coseparate $\mathcal{L}_{2}$, then, by Theorem 4.1, there exists $\mu \in I\left(\mathcal{L}_{1}\right), \nu_{1}, \nu_{2} \in I_{R}\left(\mathcal{L}_{2}\right)$ with $\nu_{1} \neq \nu_{2}$, and such that $\mu \leq \nu_{1}\left(\mathcal{L}_{1}\right)$ and $\mu \leq \nu_{2}\left(\mathcal{L}_{1}\right)$. Hence

$$
\nu_{1}=\nu_{1}^{\prime} \leq \mu^{\prime}\left(\mathcal{L}_{2}\right)
$$

and

$$
\nu_{2}=\nu_{2}^{\prime} \leq \mu^{\prime}\left(\mathcal{L}_{2}\right) .
$$

But $\mathcal{G}_{\mu^{\prime}}$ is a prime $\mathcal{L}_{2}$-filter, while $\nu_{1}$ and $\nu_{2}$ determine $\mathcal{L}_{2}$ ultrafilters. Thus, we must have $\nu_{1}=\nu_{2}$, a contradiction. Hence, $\mathcal{L}_{1}$ coseparates $\mathcal{L}_{2}$.

Next, we extend Theorem 3.5.

THEOREM 4.8. Continuing with the notation prior to and in Theorem 4.6, we have that $\mathcal{L}_{1}$ coseparates $\mathcal{L}_{2}$ if and only if $\bar{\mu}$ is submodular or, equivalently, a finitely subadditive outer measure for any $\mu \in I\left(\mathcal{L}_{1}\right)$

PROOF. 1) If $\bar{\mu}$ is submodular, then, in particular, $\bar{\mu}$ is submodular on $\mathcal{L}_{2}^{\prime}$. However, $\bar{\mu}=\mu_{2}\left(\mathcal{L}_{2}^{\prime}\right)$, so $\mu_{2}$ is submodular on $\mathcal{L}_{2}^{\prime}$. Hence $\mu^{\prime}$ is supermodular on $\mathcal{L}_{2}$. Thus if $B_{1}, B_{2} \in \mathcal{L}_{2}$, and, if $\mu^{\prime}\left(B_{1}\right)=1=\mu^{\prime}\left(B_{2}\right)$, then $\mu^{\prime}\left(B_{1} \cap B_{2}\right)=1$, and clearly $\mathcal{G}_{\mu^{\prime}}$ is a prime $\mathcal{L}_{2}$-filter, so $\mathcal{L}_{1}$ coseparates $\mathcal{L}_{2}$ by Theorem 4.7 .

2) Conversely suppose $\mathcal{L}_{1}$ coseparates $\mathcal{L}_{2}$, then by Theorem 4.6 b) $\bar{\mu}$ is a finitely subadditive outer measure for any $\mu \in I\left(\mathcal{L}_{1}\right)$.

We note if $\mathcal{L}_{1}$ coseparates $\mathcal{L}_{2}$, then $\nu=\nu^{\prime}=\mu^{\prime}\left(\mathcal{L}_{2}\right)$ since $\nu \in I_{R}\left(\mathcal{L}_{2}\right)$ and by Theorem 4.2, however, $\nu=\bar{\mu}\left(\mathcal{L}_{2}\right)$, so $\bar{\mu}=\mu^{\prime}\left(\mathcal{L}_{2}\right)$.

Suppose conversely for any $\mu \in I\left(\mathcal{L}_{1}\right), \bar{\mu}=\mu^{\prime}\left(\mathcal{L}_{2}\right)$. We note for any $E \subset X, \mu^{\prime}(E)+\mu_{2}(E)=$ $\mu(X)=1$.

If $\mathcal{L}_{1}$ does not coseparate $\mathcal{L}_{2}$, then there exists $B_{1}, B_{2} \in \mathcal{L}_{2}$ such that $B_{1} \cap B_{2}=\emptyset$, and

$$
\mathcal{H}=\left\{A^{\prime} \in \mathcal{L}_{1}^{\prime} \mid A^{\prime} \supset B_{1} \text {, or } A^{\prime} \supset B_{2}\right\}
$$

has the finite intersection property. Consequently there exists a $\mu \in\left(\mathcal{L}_{1}\right)$ such that for any $A \in \mathcal{L}_{1}$ and $A^{\prime} \supset B_{1}$ or $A^{\prime} \supset B_{2}$ then $\mu\left(A^{\prime}\right)=1$. Hence, $\mu^{\prime}\left(B_{1}\right)=\mu^{\prime}\left(B_{2}\right)=1$. Thus $\mu_{2}\left(B_{2}^{\prime}\right)=0$. But, $B_{1} \subset B_{2}^{\prime}$, so $\bar{\mu}\left(B_{1}\right)=0$, a contradiction. Therefore, $\mathcal{L}_{1}$ coseparates $\mathcal{L}_{2}$.

Summarizing, we have extended Theorem 3.6 to:

THEOREM 4.9. Using the above notation $\mathcal{L}_{1}$ coseparates $\mathcal{L}_{2}$ if and only if $\bar{\mu}=\mu^{\prime}\left(\mathcal{L}_{2}\right)$ for any $\mu \in I\left(\mathcal{L}_{1}\right)$.

\section{REFERENCES}

[1] BACHMAN, G. and SULTAN, A., Regular lattice measures: mappings and spaces, Pacific J. Math. 67, 2 (1976), 291-321.

[2] CHAN, J.K., Measures on coallocation and normal lattices, Internat. J. Math. \& Math. Sci. 15, 4 (1992), 701-718.

[3] FROLIK, Z., Prime filters with the c.i.p., Comm. Math. Univ. Caroline 13, 3 (1972), 553-573.

[4] SZETO, M., On separation of lattices, Internat. J. Math. \& Math. Sci. 14, 2 (1991), 325-338.

[5] SZETO, M., On normal lattices and separation properties of lattices, Journal of the Indian Mathematical Society 58, 1 (1992), 51-64.

[6] VLAD, C., On normal lattices and semi-separation of lattices, Journal of the Indian Mathematical Society 56 (1991), 259-273.

[7] WALLMAN, H., Lattices and topological spaces, Ann. of Math. 39 (1938), 112-126. 


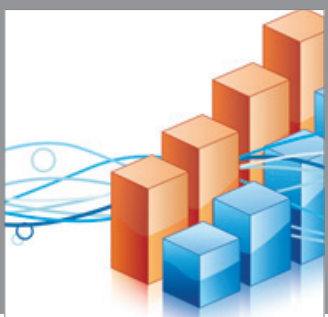

Advances in

Operations Research

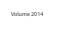

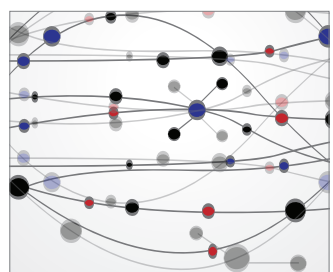

\section{The Scientific} World Journal
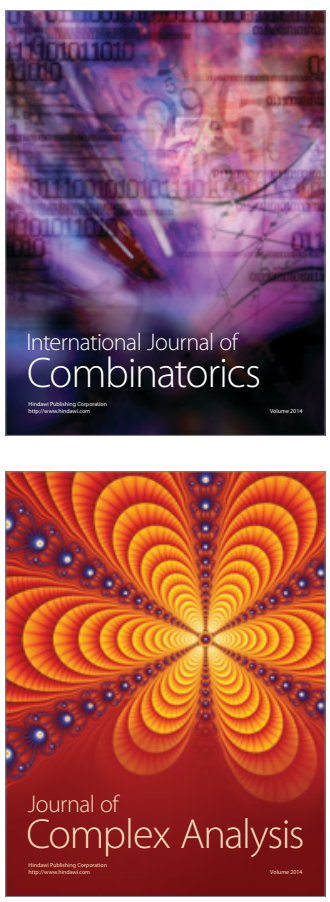

International Journal of

Mathematics and

Mathematical

Sciences
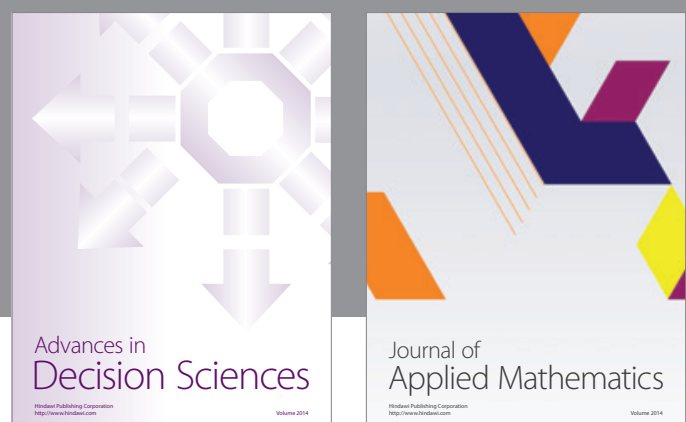

Journal of

Applied Mathematics
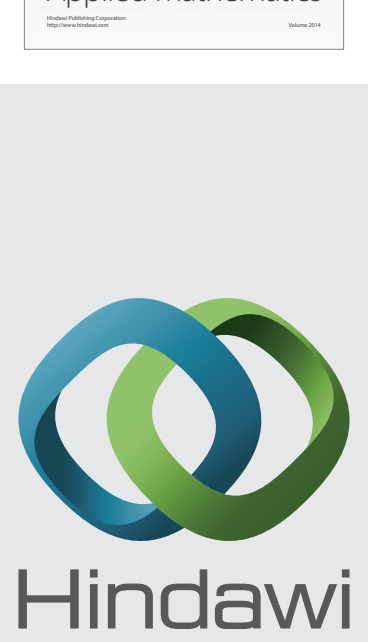

Submit your manuscripts at http://www.hindawi.com
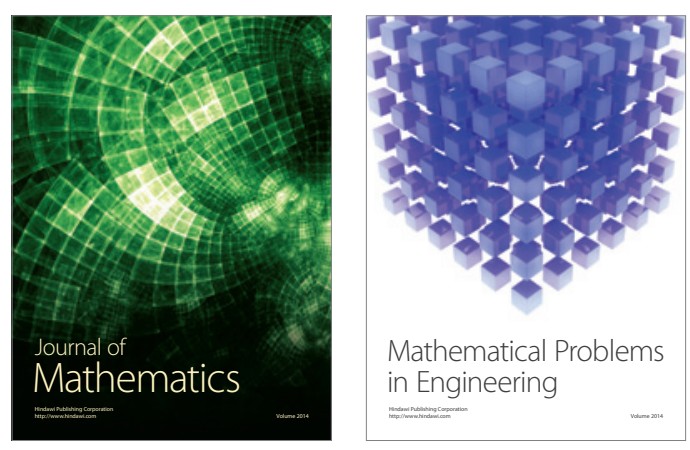

Mathematical Problems in Engineering
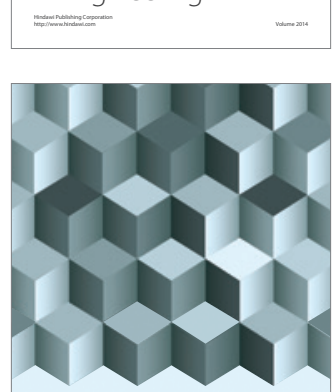

Journal of

Function Spaces
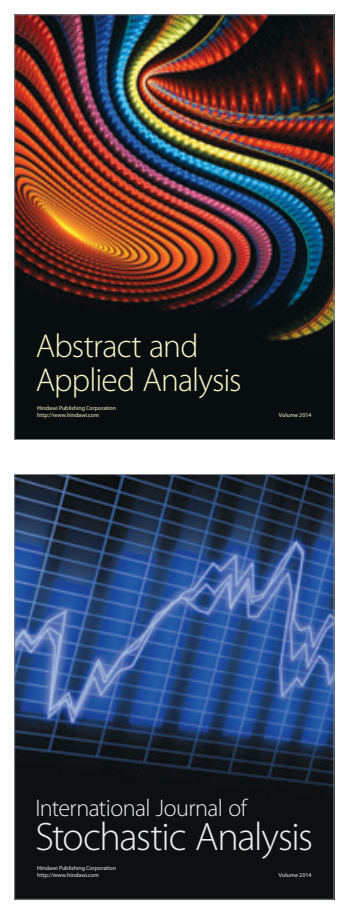

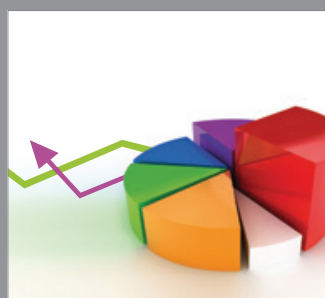

ournal of

Probability and Statistics

Promensencen
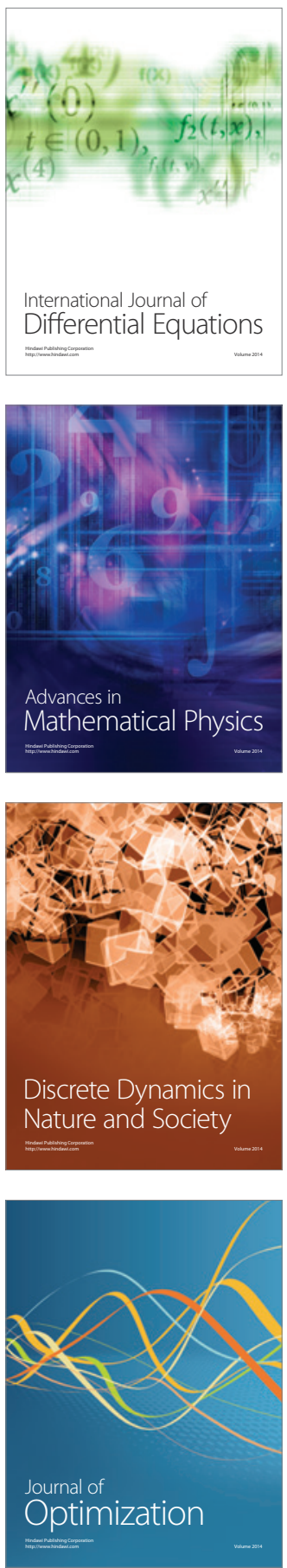